\title{
Comparison between high-field 3 Tesla MRI and computed tomography with and without arthrography for visualization of canine carpal ligaments: a cadaveric study
}

\author{
Castelli, Emanuele ; Pozzi, Antonio ; Klisch, Karl ; Scotti, Lorenza ; Hoey, Seamus ; Dennler, Matthias
}

\begin{abstract}
OBJECTIVE:To compare the quality of visualization of canine carpal ligaments by using computed tomography (CT), MRI, CT arthrography (CTA), and magnetic resonance arthrography (MRA). STUDY DESIGN: Prospective descriptive study. STUDY POPULATION: Cadavers from dogs weighing more than $20 \mathrm{~kg}$. METHODS: A 16-slice CT scanner and a 3 Tesla MRI were used for the investigation. A dilute contrast medium was injected into the middle carpal and radiocarpal joints under fluoroscopic control, and CTA and MRA images were acquired. To evaluate the difference between imaging modalities, 3 observers graded carpal ligaments of clinical interest using a scale from 0 to 4 for their quality of visualization. Data were analyzed by using a random-effect ordinal logistic regression with Bonferroni adjustment. The interobserver agreement was calculated by using the weighted Cohen's $\kappa$. RESULTS: Normal carpal joints $(n=9)$ were investigated. Magnetic resonance arthrography improved visualization of the majority of carpal ligaments compared with MRI $(\mathrm{P}<.05)$ and offered the best visualization overall. Magnetic resonance imaging and MRA offered better visualization compared with both CT and CTA $(\mathrm{P}<.05)$. There was no difference between CT and CTA. Interobserver agreement was discrete $(0.2<\kappa \leq$ 0.4 ) for all observers. CONCLUSION: Arthrography improved the capabilities of MRI but not of CT for visualization of the canine carpal ligaments. Magnetic resonance arthrography was particularly useful for evaluation of the stabilizers of the antebrachiocarpal joint. CLINICAL SIGNIFICANCE: 3 Tesla MRA and MRI allow excellent visualization of the ligamentous morphology and may be helpful in the diagnostic process of carpal sprains in dogs.
\end{abstract}

DOI: https://doi.org/10.1111/vsu.13186

Posted at the Zurich Open Repository and Archive, University of Zurich

ZORA URL: https://doi.org/10.5167/uzh-169365

Journal Article

Accepted Version

Originally published at:

Castelli, Emanuele; Pozzi, Antonio; Klisch, Karl; Scotti, Lorenza; Hoey, Seamus; Dennler, Matthias (2019). Comparison between high-field 3 Tesla MRI and computed tomography with and without arthrography for visualization of canine carpal ligaments: a cadaveric study. Veterinary Surgery, 48(4):546-555.

DOI: https://doi.org/10.1111/vsu.13186 
4 RUNNING HEAD: CT Arthrography and MR Arthrography of the canine carpus.

5 ARTICLE TITLE: Comparison between high-field 3 Tesla Magnetic Resonance Imaging

6 and Computed Tomography, with and without arthrography, for visualization of canine

7 carpal ligaments: a cadaveric study.

8 AUTHOR NAMES: Emanuele Castelli ${ }^{1}$ DVM; Antonio Pozzi ${ }^{1}$ DVM, DACVS, DECVS,

9 DACVSMR; Karl Klisch ${ }^{2}$ DVM; Lorenza Scotti ${ }^{3} \mathrm{PhD}$; Séamus Hoey ${ }^{4}$ DVM, DECVDI,

10 DACVR; Matthias Dennler ${ }^{5}$ DVM, DECVDI.

11 INSTITUTIONAL AFFILIATION: 1 - Clinic for Small Animal Surgery, Vetsuisse

12 Faculty, University of Zurich, Zurich, Switzerland. 2 - Institute of Veterinary Anatomy,

13 Vetsuisse Faculty, University of Zurich, Zurich, Switzerland. 3 - Unit of Biostatistics,

14 Epidemiology and Public Health, Department of Statistics and Quantitative Methods,

15 University of Milano-Bicocca, Milan, Italy. 4 - School of Veterinary Medicine,

16 University College Dublin, Dublin, Ireland. 5 - Clinic for Diagnostic Imaging, Vetsuisse

17 Faculty, University of Zurich, Zurich, Switzerland.

18 GRANT: This study was funded by the Stiftung für Kleintiere der Vetsuisse Fakultät

19 Universität Zürich.

20 CORRESPONDING AUTHOR: Emanuele Castelli, Clinic for Small Animal Surgery,

21 Vetsuisse Faculty, University of Zurich, CH-8057, Zurich, Switzerland, 22 ecastelli@vetclinics.uzh.ch . 
23 In submitting this manuscript, I acknowledge that the contents have been reviewed and 24 approved by all named authors.

25 This manuscript has not been submitted to, nor is under consideration by, any other 26 scientific journal for publication or on-line use.

27 Preliminary results of this study were presented at the ECVS $26^{\text {th }}$ Annual Scientific 28 Meeting 2017. 
30 OBJECTIVES: To compare the quality of visualization of canine carpal ligaments using

31 CT, MRI, Computed Tomography Arthrography (CTA), and Magnetic Resonance

32 Arthrography (MRA).

33 STUDY DESIGN: Prospective descriptive study.

34 ANIMALS: Cadavers from dogs weighing more than $20 \mathrm{~kg}$.

35 METHODS: A16-slice CT scanner and a 3 Tesla MRI were used for the investigation. A

36 dilute contrast medium was injected into the middle carpal and radiocarpal joints under

37 fluoroscopic control and CTA and MRA images were acquired. To evaluate the

38 difference between imaging modalities, three observers graded carpal ligaments of

39 clinical interest using a scale from 0 to 4 for their quality of visualization. Data were

40 analyzed using a random effect ordinal logistic regression with Bonferroni adjustment.

41 The interobserver agreement was calculated using the weighted Cohen's Kappa.

42 RESULTS: Normal carpal joints $(n=9)$ were investigated. MRA improved visualization

43 of the majority of carpal ligaments compared to MRI ( $\mathrm{p}<.05)$ and offered the best

44 visualization overall. MRI and MRA offered a better visualization than both CT and CTA

45 ( $\mathrm{p}<.05)$. There was no significant difference between CT and CTA. Interobserver

46 agreement was discrete $(0.2<$ Kappa $\leq 0.4)$ for all observers.

47 CONCLUSIONS: Arthrography improved the capabilities of MRI, but not of CT, for

48 visualization of the canine carpal ligaments. MRA was particularly useful for evaluation

49 of the stabilizers of the antebrachiocarpal joint. 
50 CLINICAL SIGNIFICANCE: 3 Tesla MRA and MRI allow excellent visualization of the

51 ligamentous morphology and may be helpful in the diagnostic process of carpal sprains in 52 dogs. 
54 Sprain injuries of the carpal ligaments are frequently encountered in dogs and can lead to 55 severe lameness and functional disability. ${ }^{1}$ Carpal sprains are sustained under a variety of 56 circumstances, such as slipping and sliding on a floor, falling or jumping from a height,

57 or tumbling over an obstacle. ${ }^{2}$ Working dogs which are exposed to repeated and sustained

58 exercise are prone to carpal sprains. ${ }^{3}$ Damage to the palmar structures leads to carpal

59 hyperextension, which is the most common type of carpal injury in the dog. ${ }^{4}$

60 The evaluation of the sprained carpus poses a diagnostic challenge given the small size 61 and large number of ligaments that stabilize the joints. Orthopedic and radiographic 62 examinations are the mainstay of the diagnostic process, and are sufficient in most of the 63 cases to achieve an accurate diagnosis. ${ }^{5,6}$ However, some patients suffer from long-lasting 64 pain and reduced carpal function due to diagnosis oversight, followed by inappropriate 65 treatments. $^{7}$ Stressed radiographs aid to better determine the level of instability, ${ }^{8}$ but 66 especially in cases of mild or moderate sprains, they may not adequately portray spatial 67 derangement of the individual joint levels. ${ }^{7}$ In people advanced imaging modalities such 68 as Magnetic Resonance Arthrography (MRA) and Computed Tomography Arthrography 69 (CTA) are routinely used to identify ligament tears when radiographs are normal. ${ }^{9}$

70 Arthrography adds to the capabilities of conventional CT and MRI by distending the joint 71 capsule, identifying contrast solution leakages, and ligament tears of the human wrist.

72 While previous studies have shown that MRI is a feasible imaging modality not only for 73 research but also for clinical use in case of carpal pathology in dogs, ${ }^{10-13}$ to the authors

74 knowledge there are no studies that reported the use of CTA and high-field 3 Tesla MRA 75 in the evaluation of the carpal ligaments of the dog. The aim of this study was (1) to 
76 evaluate if the addition of intraarticular contrast medium increases the visualization of

77 normal canine carpal ligaments compared to conventional CT and MRI techniques; (2) to

78 evaluate which imaging technique offers the best visualization of the ligaments overall;

79 and (3) to determine the interobserver agreement. We hypothesized (1) that CTA and

80 MRA would provide a better visualization than CT and MRI, respectively, and; (2) that

81 MRA would provide the best visualization overall. 


\section{Specimens}

84 Unpaired normal thoracic $(\mathrm{n}=9)$ limbs were obtained with owner permission from

85 skeletally mature dogs weighing over $20 \mathrm{~kg}$ that were euthanized for reasons unrelated to

86 the study. No clinical data relating to those animals were available. Specimens were

87 collected according to our institution regulation. Carpal joints without gross pathological

88 findings on orthopedic examination were included. The exclusion criterion was the

89 presence of ligament abnormalities or osteoarthritis in CT or MRI. Limbs were transected

90 at the level of the distal humerus and frozen at $-20^{\circ} \mathrm{C}$ until the imaging study. Limbs

91 were thawed to room temperature for 24 hours and the carpal region was clipped

92 circumferentially prior to imaging. In order to acquire images in a similar angulation

$93\left(180^{\circ}\right)$ throughout all imaging studies, limbs were mounted and held in position with tie

94 wraps on a custom-made wooden stabilization device (Figure 1).

95

96 Imaging

97 Contiguous $0.8 \mathrm{~mm}$ thick slices were acquired using a 16-slice CT scanner (Philips 16

98 Brilliance, Philips AG, Zurich, Switzerland) from the distal radial metaphysis to the

99 proximal metacarpal metaphyses in medium and high frequency reconstruction

100 algorithms. The CT settings used were: $250 \mathrm{~mA}, 130 \mathrm{kVp}$ with a rotation time of 1

101 second. An acquisition matrix of 1024x1024 was used. The field of view (FOV) was

102 adjusted to the dimensions of the specimens. Multiplanar reconstructions of the carpus

103 were generated in transverse, sagittal, and dorsal planes. 
104 MRI and MRA images were obtained using a 3.0 Tesla magnet (Philips Ingenia, Philips

105 AG, Zurich, Switzerland) and a surface coil Micro-47 (Philips Medical Systems, Best,

106 The Netherlands). Due to limb fixation maintained using the wooden device, the limb

107 positioning was equivalent to that of the CT imaging. Dependent on the size of the

108 specimen, the FOV was adjusted and $1 \mathrm{~mm}$ contiguous slices were acquired. Alignment

109 of the transverse, sagittal, and dorsal scan planes equaled CT image acquisition. MRI

110 scanning parameters are summarized in Table 1.

111 After CT and MRI images had been acquired, a diluted contrast mixture containing

112 iohexol and gadodiamide was injected with a 22-gauge needle from a dorsal approach

113 into the middle carpal joint and then into the radiocarpal joint under fluoroscopy control

114 (Allura Xper FD20 Biplane, Philips). Contrast medium concentration was based on

115 human reference values, ${ }^{14}$ and the final solution contained $175 \mathrm{mgI} / \mathrm{ml}$ iohexol

116 (Accupaque 350, GE Healthcare Buchler GmbH \& Co. KG, München, Germany) and

117 1:200 gadolinium:solution ratio (Omniscan $0.5 \mathrm{mmol} / \mathrm{ml}$, GE Healthcare). Aspiration of

118 synovial fluid before contrast injection, flow of contrast medium away from the needle

119 tip, and opacification of the joint spaces on the palmar side confirmed adequate intra-

120 articular contrast administration. Contrast injection was discontinued as soon as palpable

121 resistance was sensed during pressure on the syringe plunger or contrast backflow was

122 identified on fluoroscopy.

123 Subsequent to contrast injection, CTA and MRA images were acquired using the same

124 imaging protocol for $\mathrm{CT}$ as well as T1 weighted sequences for MRI.

126 Anatomic preparation 
127 After completion of CT, MRI, CTA and MRA image acquisition, the extremities were

128 frozen at $-80^{\circ} \mathrm{C}$ while still mounted on the fixation device for at least $24 \mathrm{~h}$. The frozen

129 joints were sliced into $2 \mathrm{~mm}$ thick sections in dorsal (3 specimens), sagittal (3

130 specimens), and transverse planes (3 specimens) using an electric band saw. Sliced

131 anatomic specimens were digitally scanned (Epson Perfection V700 Photo Scanner,

132 Epson Deutschland GmbH, Kloten, Switzerland).

133

134 Images analysis

135 CT, MRI, CTA, and MRA images were evaluated by three independent observers with

136 different levels of training, including two board-certified radiologists, and a first-year

137 surgical resident. Images were viewed using an open source imaging software (Osirix

138 version 3.9.4, 32-bit, Pixmeo, Geneva, Switzerland). Observers were permitted to alter

139 the scan plane, the window level and width, and the zoom within the individual imaging

140 study. Anatomic slices were available as anatomic reference for the observers during the

141 analysis of the digital images.

142 For each imaging modality the observers identified and scored selected carpal ligaments

143 and soft tissue structures of clinical interest (Table 2). The visibility of the ligaments was

144 graded on an integer numeric scale with scores from 0 to 4 (no (0), poor (1), satisfactory

145 (2), good (3), and excellent (4) visualization).

147 Statistical Analysis

148 The frequency of the visualization score for each individual ligament in CT, MRI, CTA, 149 and MRA was calculated to describe the performance of each of the four imaging 
150 modalities. A random effect ordinal logistic regression was applied to evaluate the 151 difference between imaging modalities. Two random effects were considered, the 152 observers and the specimens, assuming that observations within observers and specimens 153 were clustered and therefore more similar than between observers and specimens. The 154 model estimated the probability of having low visualization scores by comparing each 155 score level with the higher ones ( 0 vs $1,2,3,4 ; 0,1$ vs $2,3,4$ and so on). The analyses were 156 performed considering all the structures together (pooled data) and each individual 157 anatomical structure for all the imaging modalities. Given the large number of tests 158 performed, the Bonferroni adjustment was applied to take into account the problem of 159 multiplicity. $\mathrm{P}$ values $<.05$ were considered significant. The interobserver agreement was 160 calculated between pairs of observers using the weighted Cohen's Kappa, where the

161 discordances between adjoining categories (e.g. observer $1=$ score 3 , observer $2=$ score 162 4) had a smaller weight than distances between distant categories (e.g. observer $1=$ score

1630 , observer $2=$ score 4 ). According to the scale of Landis and Koch, the agreement was 164 classified as: no agreement $(0 \leq \mathrm{k})$, poor $(0<\mathrm{k} \leq 0.2)$, discrete $(0.2<\mathrm{k} \leq 0.4)$, moderate $(0.4$ $165<\mathrm{k} \leq 0.6)$, good $(0.6<\mathrm{k} \leq 0.8)$, great $(0.8<\mathrm{k} \leq 1.0)$. 
167 Arthrography

168 Depending on the size of the specimen, arthrography was performed using 1 to $3 \mathrm{ml}$ of

169 contrast solution injected into the radiocarpal joint and 1 to $2 \mathrm{ml}$ of contrast solution into

170 the middle carpal joint. Communication between the radiocarpal and middle carpal joint

171 was absent in all specimens. Opacification of the joint spaces on the palmar side

172 confirmed adequate contrast administration into the middle carpal joint. Opacification of

173 the dorsal and palmar capsular recesses confirmed adequate contrast administration into

174 the radiocarpal joint (Figure 2). Mild extravasation of contrast material outside the joints

175 along the needle tract occurred in some specimens, possibly as a result of overdistention

176 of the joint, but did not interfere with the evaluation of the images.

178 Comparison of techniques considering all the ligaments together (pooled data)

179 The frequencies of the visualization scores according to the imaging technique on the

180 overall sample are presented in Figure 3. MRA had the highest frequency of score 4 181 (45\%) compared to the other imaging techniques, and had a lower frequency of score 0 $182(3.4 \%)$ and $1(8 \%)$ compared to MRI (score $0=6.1 \%$; score $1=12.5 \%$ ). MRI had a 183 higher frequency of score $3(30 \%)$ and 4 (34\%) compared to both CTA (score $3=21 \%$; 184 score $4=2.1 \%$ ) and CT (score $3=21.6 \%$; score $4=3.2 \%$ ).

185 The results of the regression analysis are presented in Table 3. MRA demonstrated a 186 better visualization of the ligaments compared to MRI ( $\mathrm{p}<.05)$. MRI and MRA offered a 187 better visualization compared to both CT and CTA ( $\mathrm{p}<.05)$. There was no difference 188 between CT and CTA. 
190 Comparison of techniques considering each individual ligament

191 The frequencies of the visualization scores according to the imaging technique for each

192 individual ligament are presented in Figure 4. For all anatomical structures the frequency

193 of score 3 or 4 were higher for MRI and MRA compared to CT and CTA. No strong

194 differences were observed between CT and CTA. Conversely, for several anatomical

195 structures such as the palmar radiocarpal and ulnocarpal ligaments, the frequency of score

1964 were higher in MRA than MRI.

197 The results of the regression analysis are presented in Table 4. MRA significantly

198 improved the visualization of all ligaments compared to MRI ( $<$ <.05), excluding the

199 dorsal radiocarpal ligament, the lateral accessoriometacarpal ligament, the medial 200 accessoriometacarpal ligament, and the palmar fibrocartilage, where no difference was 201 found. Regarding CT and CTA, no differences were found in any anatomical structures.

202 Representative images that show the difference between CT, CTA, MRI, and MRA are 203 presented (Figures 5,6,7).

205 Interobserver agreement

206 The interobserver agreement was discrete for all observers, with weighted Kappa = 2070.3079 for radiologist number 1 vs surgery resident comparison; weighted Kappa = 2080.2574 for radiologist number 2 vs surgery resident comparison; and weighted Kappa $=$ 2090.2874 for radiologist number 1 vs radiologist number 2 comparison. 
211 This is the first study that describes the use of CTA and 3 Tesla MRA in the evaluation of

212 the carpal ligaments of the dog. Our results suggest that MRA is superior to MRI, CTA

213 and CT in the visualization of the intact carpal ligaments of dogs weighing more than 20

$214 \mathrm{~kg}$. MRA was particularly useful in the evaluation of the stabilizers of the

215 antebrachiocarpal joint, such as the palmar ulnocarpal and radiocarpal ligaments, the

216 radioulnar ligament, and the medial and lateral collateral ligaments. Contrast medium

217 introduction increased intraarticular pressure and joint capsule distension, allowing easier

218 recognition of the edges of the collateral ligaments, as well as surrounding the

219 intraarticular palmar ulnocarpal and radiocarpal ligaments. Furthermore, the presence of

220 contrast medium intensified the contrast-to-noise ratio, offering a better visualization also

221 for some extra-articular structures such as the tendon of the musculus flexor carpi ulnaris.

222 These findings are in agreement with previous studies that described the benefits of MRA

223 in the visualization of intraarticular structures, such as the cruciate ligaments of the stifle

224 joint, ${ }^{15-16}$ and the biceps tendon of the shoulder joint in dogs. ${ }^{17}$ Conversely, these finding

225 are in contrast to published literature in human medicine, where CTA is considered as

226 accurate or even more accurate than conventional 3 Tesla MRI for detecting tears of the

227 intrinsic ligaments, such as the scapholunate and lunotriquetral ligaments. ${ }^{18}$ Tears of

228 these ligaments typically manifest in CTA with contrast filling of the defect and abnormal

229 communication between the different joint compartments. Further studies may evaluate if

230 the diagnostic value of CTA may be improved in cases of tears of the intercarpal

231 ligaments in dogs. 
232 Due to its high contrast resolution, high-field 3 Tesla MRI facilitated excellent 233 visualization of the ligamentous morphology. For some anatomic structures such as the 234 lateral and medial accessoriometacarpal ligaments and palmar carpal fibrocartilage, MRA

235 did not provide a better visualization compared to MRI. The accessoriometacarpal 236 ligaments and the palmar fibrocartilage are major contributors to prevention of carpal 237 hyperextension, ${ }^{19}$ which is the most common type of carpal injury in the dog. These large 238 extra-articular structures could be clearly and consistently identified in MRI, suggesting 239 that the major palmar stabilizers of the carpus can be characterized with MRI without 240 arthrography.

241 CTA showed poor soft tissue contrast resolution, allowing only indirect recognition of the 242 edges of the ligaments and did not result in a significantly better visualization compared 243 to CT. This is in contrast to previous studies that have reported an improved visibility of 244 intraarticular ligaments of the canine stifle and shoulder joints with CTA. ${ }^{20-21}$ A possible 245 explanation could be the smaller joint dimension and tightness of the carpus compared to 246 bigger joints, and the associated inherent difficulties of joint distension of the carpal joint.

247 Furthermore, only few carpal ligaments are fully intraarticular, while the cruciate 248 ligaments of the stifle and the biceps tendon of the shoulder are contained within the 249 joint, allowing better contrast distribution around these structures.

250 CT has an inherent poor soft tissue contrast resolution compared to MRI, but we included 251 the CT data in the analysis in order to compare pre- and post arthrography CT studies and 252 to determine if CTA could improve the identification of the canine carpal ligaments.

253 There are several limitations to this study. First, the high-field strength of the 3 Tesla 254 MRI machine may have affected the comparison between the different imaging 
255 techniques. Inclusion of a low-field MRI in the study could have helped to determine the

256 impact of the magnetic field strength on the results. However, one of the purposes was to

257 test if addition of intraarticular contrast could increase the visualization of ligaments

258 compared to conventional CT and MRI. This evaluation was independent from the field-

259 strength of the MRI machine and did not affect the results of the comparison between the

260 conventional techniques and the arthrography techniques.

261 Second, the qualitative assessment performed by visual evaluation and scoring of the

262 ligaments may have been susceptible to inaccuracy due to subjective perspective of the

263 observers. A quantitative assessment by measuring relative signal intensity and relative

264 contrast of the ligaments on digital images has been described, ${ }^{22}$ and could have been a

265 more objective methodology. However, the clinical and practical utility of the individual

266 imaging modality was questioned and as such the qualitative assessment of the observers

267 was investigated.

268 Third, we found only a discrete $(0.2<$ Kappa $\leq 0.4)$ interobserver agreement. Since our

269 study was the first one to assess the carpal ligaments with MRA and CTA, we decided to

270 determine the interobserver agreement and include at least two board-certified

271 radiologists to minimize the degree of subjective interpretation. The lack of previous

272 studies and limited experience with arthrography of the carpus likely affected the

273 interobserver agreement. In support to this assumption, the interobserver agreement

274 between the two board-certified radiologist was not substantially different compared to

275 the interobserver agreement between the surgery resident and each board-certified

276 radiologist. The choice to include one resident in the analysis was driven by the

277 consideration that evaluation of diagnostic images is performed frequently by observers 
278 with different level of experience, including residents. Further training and experience

279 with the arthrography technique may improve identification of the ligaments and

280 interobserver agreement.

281 Another limitation of the study is that the volume of contrast medium and injection

282 pressure was not standardized among specimens. This variability may have affected the

283 results, with insufficient or excessive contrast medium decreasing the value of MRA and

284 CTA due to inadequate contrast distribution or artefacts due to extra-articular contrast

285 leakage artefacts. However, contrast injection was performed using manual pressure

286 control by the same operator and appropriate joint filling was confirmed with

287 fluoroscopy. The type of contrast medium was chosen based on recommendations

288 available for people and effects of varying volumes and concentrations of contrast

289 medium were beyond the scope of this study.

290 Finally, a limitation of this study is represented by its ex-vivo nature. Freezing and

291 thawing of the specimens and specimen variability may have affected the quality of

292 visualization of the ligaments and the results of the study. Further investigations

293 conducted on a more homogeneous in-vivo population would be advantageous. The use

294 of cadaveric specimens could also have affected the capacity of joint distension and

295 subsequently the volume of contrast medium injected, which may be altered in dogs with

296 carpal pathology and joint effusion. Joint effusion may yield an "arthrogram-like" effect

297 in T2 sequences, sufficient to eliminate the need for intraarticular contrast in a clinical

298 scenario. Furthermore, the cadaveric study design prevented assessment of the impact of

299 intravenous administration of contrast medium, which could show changes in signal

300 intensity and have great diagnostic value in a clinical scenario. 
301 In summary, this study supports the use of high-field 3 Tesla MRI and MRA for 302 visualization of the canine carpal ligaments. MRA improved significantly the 303 visualization of the majority of ligaments, and was particularly useful in the evaluation of

304 the stabilizers of the antebrachiocarpal joint, while the major palmar stabilizers of the 305 carpus could be identified in MRI without arthrography. These techniques may be useful

306 for identification of specific ligament injuries and for guiding treatment selection in

307 canine patients, such as selecting candidates for ligament reconstruction and for partial or 308 pancarpal arthrodesis. However, the usefulness of these techniques has first to be

309 validated in clinical patients where other factors such as joint effusion, joint fibrosis and 310 osteoarthrosis may affect visibility of the ligamentous structures. 
312 None of the listed authors have a financial interest in any of the products or companies 313 described in this manuscript. 
315 The authors would like to thank Mr. Urs Müller for the assistance in the preparation of

316 the anatomic specimens and Mr. Pascal Glatzfelder for the assistance in the image

317 editing. 
319 1. Earley TD, Dee JF. Trauma to the carpus, tarsus, and phalanges of dogs and cats.

$320 \quad$ Vet Clin North Am Small Anim Pract. 1980;10:717-47.

321 2. Earley T. Canine carpal ligament injuries. Vet Clin North Am. 1978;8:183-99.

322 3. Iddon J, Lockyer RH, Frean SP. The effect of season and track condition on injury 323 rate in racing greyhounds. J Small Anim Pract. 2014;55:399-404.

324 4. Kapatkin AS, Garcia-Nolen T, Hayash IK. Carpus, metacarpus, and digits. In:

325 Tobias KM, Johnston, SA, editors. Veterinary Surgery Small Animal. 1st edn. $326 \quad$ Saunders/Elsevier St. Louis, 2012:785-800.

327 5. Farrow CS. Stress radiography: applications in small animal practice. $J$ Am Vet $328 \quad$ Med Assoc. 1982;181:777-784.

329 6. Langley-Hobbs SJ, Hamilton MH, Pratt JN. Radiographic and clinical features of 330 carpal varus associated with chronic sprain of the lateral collateral ligament 331 complex in 10 dogs. Vet Comp Orthop Traumatol. 2007;20:324-330.

332 7. Farrow CS. Carpal sprain injury in the dog. J Am Vet Rad Soc. 1977;18:38-44.

333 8. Piermattei DL, Flo GL, DeCamp CE. Fractures and other orthopedic conditions of 334 the carpus, metacarpus and phalanges. In: Piermattei DL, Flo GL, DeCamp CE, 335 editors. Brinker, Piermattei, and Flo's handbook of small animal orthopedics and 336 fracture repair. 4th edn. Saunders/Elsevier, St Louis, 2006:382-412.

337 9. Ramamurthy NK, Chojnowski AJ, Toms AP. Imaging in carpal instability. $J$ $338 \quad$ Hand Surg Eur. 2017; 41:22-34.

339 10. Nordberg CC, Johnson KA. Magnetic resonance imaging of normal canine carpal $340 \quad$ ligaments. Vet Radiol Ultrasound. 1999;40:128-36. 
11. Pownder SL, Cooley S, Hayashi K, Bezuidenhout A, Koff MF, Potter HG.

342 Non-invasive magnetic resonance imaging diagnosis of presumed 343 intermedioradial carpal bone avascular necrosis in the dog. Can Vet $J$. $344 \quad 2016 ; 57: 879-81$.

345 12. Porter IR, Pownder SL, McDonough SP, Potter HG, Hayashi K. Nonarticular 346 osseous cyst-like lesions of the intermedioradial carpal bone may be incidental 347 magnetic resonance imaging findings in dogs. Vet Radiol Ultrasound. $348 \quad 2018 ; 59: 715-720$.

349 13. Basa RM, Crowley AM, Johnson KA. Neurofibroma of the ulnar nerve in the 350 carpal canal in a dog: treatment by marginal neurectomy. [Epub ahead of print 19 351 Oct 2018]. J Small Anim Pract. doi: 10.1111/jsap.12945.

352 14. Wyler A, Bousson V, Bergot C, et al. Comparison of MR-arthrography and CT353 arthrography in hyaline cartilage-thickness measurement in radiographically 354 normal cadaver hips with anatomy as gold standard. Osteoarthr Cartil. $355 \quad 2009 ; 17: 19-25$.

356 15. Banfield CM, Morrison WB. Magnetic resonance arthrography of the canine stifle 357 joint: technique and applications in eleven military dogs. Vet Radiol Ultrasound. $358 \quad 2000 ; 41: 200-213$.

359 16. Pujol E, Van Bree H, Cauzinille L, Poncet C, Gielen I, Bouvy B. Anatomic study 360 of the canine stifle using low-field magnetic resonance imaging (MRI) and MRI $361 \quad$ arthrography. Vet Surg. 2011;40:395-401.

362 17. De Rycke LM, Gielen IM, Dingemanse W, et al. Computed Tomographic and 363 Low-Field Magnetic Resonance Arthrography: a comparison of techniques for 
observing intra-articular structures of the normal canine shoulder. Vet Surg. $2015 ; 44: 704-12$.

18. Lee RKL, Ng AWH, Tong CSL, Griffith JF, Tse WL, Wong C et al. Intrinsic ligament and triangular fibrocartilage complex tears of the wrist: comparison of MDCT arthrography, conventional 3-T MRI, and MR arthrography. Skelet Radiol. 2013;42:1277-1285.

19. Shetye SS, Malhotra K, Ryan SD, Puttlitz CM. Determination of mechanical properties of canine carpal ligaments. Am J Vet Res. 2009;70:1026-30.

20. Samii VF, Dyce J. Computed tomographic arthrography of the normal canine stifle. Vet Radiol Ultrasound. 2004;45:402-406.

374 21. Eivers CR, Corzo-Menéndez N, Austwick SH, et al. Computed tomographic arthrography is a useful adjunct to survey computed tomography and arthroscopic evaluation of the canine shoulder joint. Vet Radiol Ultrasound. 2018;59:535-544. 
381 Figure 1. A custom-made wooden stabilization device was used to hold the limbs in the 382 same position throughout the imaging study.

384 Figure 2. Fluoroscopic images, lateral view. A, Contrast is injected with a 22-gauge 385 needle from a dorsal approach into the middle carpal joint. B, Opacification of the joint 386 spaces on the palmar side confirms adequate contrast administration into the middle 387 carpal joint (arrowhead). Please note the absence of communication between the 388 radiocarpal and middle carpal joint. C, Contrast is injected into the radiocarpal joint. D, 389 Opacification of the dorsal and palmar capsular recesses confirms adequate contrast 390 administration into the radiocarpal joint (arrowheads).

392 Figure 3. Graphic illustrating the frequencies of the visualization scores according to the 393 imaging technique on the overall sample (pooled data).

395 Figure 4. Graphics illustrating the frequencies of the visualization scores according to the 396 imaging technique for each individual ligament.

398 Figure 5. Transverse section of the canine carpus. The medial aspect of the joint is on the 399 right of the images, and dorsal is at the top of the images. A, T1W MRI; B,T1W MRA;

$400 \mathrm{C}$, frozen anatomic section; D, T2W MRI; E, PDW MRI with fat saturation. The 401 delineation of the intraarticular ligaments such as the palmar ulnocarpal (PUCL) and the 402 palmar radiocarpal ligament (PRCL) in MRI depends on the signal characteristics of the 
403 ligaments and the synovial fluid, and is low in T1W MRI (Figure 5A) compared to T2W

404 or PDW MRI images (Figure 5D,E). Please note how arthrography facilitates recognition

405 of intraarticular ligaments by altering the signal characteristics and distension of the joint

406 (Figure 5B). R, radius; U, ulna; MECR, musculus extensor carpi radialis tendon; MAPL,

407 musculus abductor pollicis longus tendon; oMCL, oblique part of the medial collateral

408 ligament; MFCR, musculus flexor carpi radialis tendon; MFDP, musculus flexor digitalis

409 profundus tendon; MFDS, musculus flexor digitalis superficialis tendon; MFCU,

410 musculus flexor carpi ulnaris tendon; PRCL, palmar radiocarpal ligament; PUCL, palmar

411 ulnocarpal ligament; MECU, musculus extensor carpi ulnaris tendon; RUL, radioulnar

412 ligament; MEDL, musculus extensor digitalis lateralis; MEDC, musculus extensor

413 digitalis communis tendon.

415 Figure 6. Dorsal section of the canine carpus. The medial aspect of the joint is to the left 416 of the images, and proximal is at the top of the images. A, T1W MRI; B, T1W MRA; C,

417 frozen anatomic section; D, CT; E, CTA. Intraarticular contrast medium improved 418 delineation of the margins of the oblique part of the medial collateral ligament (oMCL) 419 and radioulnar ligament (RUL) in MRA (Figure 6B) compared to T1W MRI (Figure 6A). 420 CT and CTA images are presented (Figure 6D,E) in soft tissue reconstruction with soft 421 tissue windowing, optimized for delineation of ligamentous structures (WL/WW:

422 45/180). CT and CTA did not allow separation of the different components of the medial

423 collateral ligament, and delineation of the radioulnar ligament (RUL) was poor in both

424 CT and CTA. R, radius; RCB, radial carpal bone; UCB, ulnar carpal bone; CII, second 425 carpal bone; CIII, third carpal bone; CIV, fourth carpal bone; RUL, radioulnar ligament; 
426 ICL, intercarpal ligament; oMCL, oblique part of medial collateral ligament; MAPL,

427 musculus abductor pollicis longus tendon.

429 Figure 7. Sagittal section of the canine carpus. The dorsal aspect of the joint is to the left 430 of the images, and proximal is at the top of the images. A, T1W MRI; B, T1W MRA; C,

431 frozen anatomic section; D, T2W MRI; E, PDW MRI with fat saturation. Please note how

432 contrast medium allows improved delineation of the intraarticular structures, such as the

433 dorsal radiocarpal ligament (DRCL), the palmar radiocarpal (PRCL), and palmar

434 ulnocarpal ligaments (PUCL) in MRA (Figure 7B) compared to MRI (Figure 7A) due to 435 joint distension. Extra-articular structures such as the medial accessoriometacarpal 436 ligament (MACBL) are clearly visible in MRI, regardless of the MRI sequence and 437 contrast administration. $\mathrm{R}$, radius; $\mathrm{UCB}$, ulnar carpal bone; $\mathrm{ACB}$, accessory carpal bone; 438 CIV, fourth carpal bone; DRCL, dorsal radiocarpal ligament; MFCU, musculus flexor 439 carpi ulnaris tendon; AUCL, accessorioulnocarpal ligament; MACBL, medial 440 accessoriometacarpal ligament; PRCL, palmar radiocarpal ligament; PUCL, palmar 441 ulnocarpal ligament. 
444 Table 1. Summary of MRI scanning parameters used in the study.

\begin{tabular}{|c|c|c|c|c|c|c|c|c|c|}
\hline $\begin{array}{c}\text { Paramet } \\
\text { ers }\end{array}$ & \multicolumn{3}{|c|}{ T2W TSE } & \multicolumn{3}{c|}{ PDW FS } & \multicolumn{3}{c|}{ T1W } \\
\hline Plane & sag & dor & trans & sag & dor & trans & sag & dor & trans \\
\hline $\begin{array}{c}\text { ST } \\
(\mathrm{mm})\end{array}$ & 1 & 1 & 1 & 1 & 1 & 1 & 1 & 1 & 1 \\
\hline Matrix & $\begin{array}{c}228 \times 1 \\
72\end{array}$ & $\begin{array}{c}280 \times 2 \\
72\end{array}$ & $\begin{array}{c}200 \times 1 \\
69\end{array}$ & $\begin{array}{c}400 \times 2 \\
36\end{array}$ & $\begin{array}{c}400 \times 2 \\
34\end{array}$ & $\begin{array}{c}300 \times 1 \\
44\end{array}$ & $\begin{array}{c}320 \times 1 \\
68\end{array}$ & $\begin{array}{c}320 \times 1 \\
70\end{array}$ & $\begin{array}{c}200 \times 1 \\
65\end{array}$ \\
\hline TR (ms) & 2723 & 3251 & 5990 & 3088 & 3070 & 5526 & 632 & 742 & 739 \\
\hline TE (ms) & 80 & 80 & 80 & 30 & 30 & 30 & 11 & 11 & 11 \\
\hline $\begin{array}{c}\text { IG } \\
(\mathrm{mm})\end{array}$ & 0.1 & 0.1 & 0.1 & 0.1 & 0.1 & 0.1 & 0.1 & 0.1 & 0.1 \\
\hline NSA & 5 & 3 & 4 & 2 & 4 & 4 & 4 & 6 & 4 \\
\hline $\begin{array}{c}\text { FA } \\
(\text { degree })\end{array}$ & 90 & 90 & 90 & 90 & 90 & 90 & 90 & 90 & 90 \\
\hline
\end{tabular}

445 ST, slice thickness; TR, repetition time; TE, echo time; IG, interslice gap; NSA, number

446 of signal averages; FA, flip angle; T2W TSE, T2-weighted turbo spin echo; PDW FS,

447 proton density-weighted fat saturated; T1W, T1 weighted. 
448 Table 2. Ligaments and soft tissue structures selected for the investigation and relative 449 abbreviations.

\begin{tabular}{|c|c|}
\hline 1. Dorsal radiocarpal ligament (DRCL) & 450 \\
\hline \multicolumn{2}{|c|}{ 2. Insertion of the M. extensor carpi ulnaris tendon (MECW\$1 } \\
\hline \multicolumn{2}{|c|}{ 3. Insertion of the M. flexor carpi ulnaris tendon (MFCU) 452} \\
\hline 4. Lateral accessoriometacarpal ligament (LACBL) & 453 \\
\hline 5. Lateral collateral ligament (LCL) & 454 \\
\hline 6. Accessorioulnocarpal ligament (AUCL) & 455 \\
\hline 7. Medial accessoriometacarpal ligament (MACBL) & 456 \\
\hline \multicolumn{2}{|c|}{ 8. Oblique part of the short medial collateral ligament (oMASZ) } \\
\hline \multicolumn{2}{|c|}{ 9. Straight part of the short medial collateral ligament (sM 48 ) } \\
\hline 10. Palmar radiocarpal ligament (PRCL) & 459 \\
\hline 11. Palmar ulnocarpal ligament (PUCL) & 460 \\
\hline 12. Palmar carpal fibrocartilage (PCFC) & 461 \\
\hline 13. Radiocarpal-metacarpal ligament (RCML) & 462 \\
\hline 14. Radioulnar ligament (RUL) & 463 \\
\hline
\end{tabular}


465 Table 3. The estimate (beta), the corresponding standard error (S.E.) and the Bonferroni

466 adjusted $\mathrm{p}$ value on the overall sample. A negative estimate implies that the first

467 technique leads to a better visualization compared to the second technique. $\mathrm{P}<.05$ is

468 significant.

\begin{tabular}{|c|c|c|c|}
\hline Technique & beta & S.E. & $\mathrm{p}$ value \\
\hline CT vs. CTA & -0.05053 & 0.1289 & 1 \\
\hline CT vs. MRA & 7.7198 & 0.3398 & $<.0001$ \\
\hline CT vs. MRI & 17.8338 & 0.9013 & $<.0001$ \\
\hline CTA vs. MRA & 7.8714 & 0.3395 & $<.0001$ \\
\hline CTA vs. MRI & 18.2886 & 0.9003 & $<.0001$ \\
\hline MRA vs. MRI & -5.3256 & 0.6074 & $<.0001$ \\
\hline
\end{tabular}


470 Table 4. The estimate (beta), the corresponding standard error (S.E.) and the Bonferroni

471 adjusted $\mathrm{p}$ value for the single structures. A negative estimate implies that the first

472 technique leads to a better visualization compared to the second technique. $\mathrm{P}<.05$ is

473 significant.

\begin{tabular}{|c|c|c|c|c|c|c|}
\hline \multirow{2}{*}{ Anatomical } & \multicolumn{3}{|c|}{ MRA vs MRI } & \multicolumn{3}{c|}{ CTA vs CT 474} \\
\cline { 2 - 7 } & beta & S.E. & p value & beta & S.E. & p value \\
\hline DRCL & -4.7 & 2.1 & 0.05 & 0.1 & 0.5 & 1 \\
\hline MECU & -10.3 & 3.4 & $<.05$ & 0.2 & 0.4 & 1 \\
\hline MFCU & -6.8 & 2.3 & $<.05$ & -0.2 & 0.4 & 1 \\
\hline LACBL & 3.4 & 2.8 & 0.4 & 0.3 & 0.5 & 1 \\
\hline LCL & -5.5 & 2.4 & $<.05$ & 0.3 & 0.4 & 0.9 \\
\hline AUCL & -9.8 & 2.2 & $<.05$ & 0.01 & 0.6 & 1 \\
\hline MACBL & 5.1 & 2.6 & 0.1 & 0.09 & 0.5 & 1 \\
\hline oMCL & -10.4 & 0.3 & $<.05$ & 0.4 & 0.7 & 1 \\
\hline sMCL & -5.9 & 2.2 & $<.05$ & 0.3 & 0.5 & 0.8 \\
\hline PRCL & -14.7 & 2.3 & $<.05$ & -0.9 & 0.4 & 0.1 \\
\hline PUCL & -23.5 & 3.0 & $<.05$ & 0.02 & 0.5 & 1 \\
\hline PCFC & -1.6 & 2.2 & 0.9 & 0.07 & 0.5 & 1 \\
\hline RCMCL & -11.0 & 4.0 & $<.05$ & 0.7 & 0.5 & 0.3 \\
\hline RUL & -8.8 & 2.4 & $<.05$ & -0.5 & 0.5 & 0.6 \\
\hline
\end{tabular}

